\title{
What are the essential components of Antenatal Care? A systematic review of the literature and development of signal functions to guide monitoring and evaluation.
}

\author{
Hannah McCauley ${ }^{1}$, Kirsty Lowe ${ }^{1}$, Nicholas Furtado ${ }^{2}$, Viviana Mangiaterra ${ }^{3}$, and Nynke \\ van den Broek ${ }^{1}$ \\ ${ }^{1}$ Centre for Maternal and Newborn Health \\ ${ }^{2}$ The Global Fund to Fight AIDS Tuberculosis and Malaria \\ ${ }^{3}$ Bocconi University
}

August 9, 2021

\begin{abstract}
Background Antenatal Care (ANC) is one of the key care-packages required to reduce global maternal and perinatal mortality and morbidity Objectives To identify the essential components of ANC and develop signal functions Search strategy MESH headings for databases including Cinahl, Cochrane, Global Health, Medline, PubMed, and Web of Science Selection Criteria Papers and reports on content of ANC published from 2000-2020 Data collection and Analysis Narrative synthesis of data and development of signal function through 7 consensus-building workshops with 184 stakeholders Main Results A total of 221 papers and reports are included from which 28 essential components of ANC were extracted and used to develop 15 signal functions with the equipment, medication and consumables required for implementation of each. Signal functions for the prevention and management of infectious diseases (malaria, HIV, tuberculosis, syphilis and tetanus) can be applied depending on population disease burden. Screening and management of pre-eclampsia, gestational diabetes, anaemia, mental and social health (including intimate partner violence) are recommended universally. Three signal functions adress monitoring of foetal growth and wellbeing and identification and management of obstetric complications. Promotion of health and wellbeing via education and support for nutrition, cessation of substance abuse, uptake of family planning, recognition of danger signs and birth preparedness are included as essential components of ANC. Conclusions New signal functions have been developed which can be used for monitoring and evaluation of content and quality of ANC. Country adaptation and validation is recommended.
\end{abstract}

\section{Hosted file}

Main manuscript - Systematic Review ANC and development of signal functions..docx available at https://authorea.com/users/429781/articles/533410-what-are-the-essentialcomponents-of-antenatal-care-a-systematic-review-of-the-literature-and-development-ofsignal-functions-to-guide-monitoring-and-evaluation 